PROCEEDINGS OF THE

AMERICAN MATHEMATICAL SOCIETY

Volume 126, Number 1, January 1998, Pages 115-121

S $0002-9939(98) 03839-8$

\title{
MALUTA'S COEFFICIENT \\ IN MUSIELAK-ORLICZ SEQUENCE SPACES EQUIPPED WITH THE ORLICZ NORM
}

\author{
YUNAN CUI, HENRYK HUDZIK, AND HONGWEI ZHU
}

(Communicated by Palle E. T. Jorgensen)

\begin{abstract}
Maluta's coefficient of Musielak-Orlicz sequence spaces equipped with the Orlicz norm is calculated. A sufficient condition for the Schur property of these spaces is given.
\end{abstract}

\section{INTRODUCTION}

In the sequel $\mathbb{N}, \mathbb{R}$ and $\mathbb{R}_{+}$stand for the set of natural numbers, the set of reals and the set of nonnegative reals, respectively. The space of all sequences $x=(x(i))_{i=1}^{\infty}$ of reals is denoted by $l^{0}$. A map $\Phi: \mathbb{R} \rightarrow[0,+\infty)$ is said to be an Orlicz function if $\Phi$ is convex, even, vanishing at zero, left continuous on $\mathbb{R}_{+}$and not identically equal to zero (see $[9,12,13,15]$ and [16]).

A sequence $\Phi=\left(\Phi_{i}\right)_{i=1}^{\infty}$ of Orlicz functions $\Phi_{i}$ is called a Musielak-Orlicz function (see [15]). By $\Psi=\left(\Psi_{i}\right)$ we denote the Musielak-Orlicz function conjugate to $\Phi=\left(\Phi_{i}\right)$ in the sense of Young, i.e.

$$
\Psi_{i}(u)=\sup _{v>0}\left\{|u| v-\Phi_{i}(v)\right\}
$$

for each $u \in \mathbb{R}$ and $i \in \mathbb{N}$. Further, $\varphi=\left(\varphi_{i}\right)$ is the right derivative of $\Phi=\left(\Phi_{i}\right)$, i.e. $\varphi_{i}$ are the right derivatives of $\Phi_{i}$ for every $i \in \mathbb{N}$.

Given a Musielak-Orlicz function $\Phi=\left(\Phi_{i}\right)$ we define on $l^{0}$ a convex modular $I_{\Phi}$ by

$$
I_{\Phi}(x)=\sum_{i=1}^{\infty} \Phi_{i}\left(x_{i}\right) \quad\left(\forall x=\left(x_{i}\right) \in l^{0}\right),
$$

and the Musielak-Orlicz space $l^{\Phi}$ by

$$
l^{\Phi}=\left\{x \in l^{0}: I_{\Phi}(\lambda x)<+\infty \text { for some } \lambda>0\right\} .
$$

Received by the editors July 19, 1995 and, in revised form, March 12, 1996.

1991 Mathematics Subject Classification. Primary 46E30.

Key words and phrases. Schur's property, Maluta's coefficient, asymptotic equidistant sequence, reflexivity, weak convergence, the $\delta_{2}$-condition.

The first and third authors were supported by the Chinese National Science Foundation.

The second author was supported by KBN grant 2 P03A 03110.

(C) 1998 American Mathematical Society 
The functional

$$
\|x\|=\sup \left\{\sum_{i=1}^{\infty} x(i) y(i): I_{\Psi}(y) \leq 1\right\}
$$

is a norm in $l^{\Phi}$ (called the Orlicz norm) and the couple $\left(l^{\Phi},\|\|\right)$ is a Banach space (see [15]).

Our aim in this paper is to calculate Maluta's coefficient of Musielak-Orlicz spaces equipped with the Orlicz norm. This coefficient is connected with normal structure, which is a very important property of Banach spaces that guarantees the fixed point property for them (see [1, 2, 3, 4, 6, 8, 10, 11, 14] and [19]).

Let in the sequel $X$ denote a reflexive infinite dimensional Banach space (which automatically does not have the Schur property) and let $S(X)$ denote its unit sphere. For each sequence $\left(x_{n}\right)$ in $X$, we define

$$
\begin{aligned}
A\left(\left(x_{n}\right)\right) & =\lim _{n}\left\{\sup \left\{\left\|x_{i}-x_{j}\right\|: i \neq j ; i, j \geq n\right\}\right\}, \\
A_{1}\left(\left(x_{n}\right)\right) & =\lim _{n}\left\{\inf \left\{\left\|x_{i}-x_{j}\right\|: i \neq j ; i, j \geq n\right\}\right\} .
\end{aligned}
$$

The weak uniform normal structure coefficient of $X$ is defined by (see [4])

$W C S(X)=\sup \left\{k>0\right.$ : for each weakly convergent sequence $\left(x_{n}\right)$ in $S(X)$ there is some $y \in \operatorname{conv}\left(x_{n}\right)$ such that

$$
\left.k \limsup \left\|x_{n}-y\right\| \leq A\left(\left(x_{n}\right)\right)\right\} \text {. }
$$

A sequence $\left(x_{n}\right)$ in $X$ is said to be an asymptotic equidistant sequence if $A\left(\left(x_{n}\right)\right)$ $=A_{1}\left(\left(x_{n}\right)\right)$. This definition was introduced in [19], where it was proved that $W C S(X)=\inf \left\{A\left(\left(x_{n}\right)\right):\left(x_{n}\right)\right.$ is an asymptotic equidistant sequence in $S(X)$ and $x_{n} \rightarrow 0$ weakly $\}$.

Recall that Maluta's coefficient $M(X)$ of a Banach space $X$ is defined by (see [14])

$$
M(X)=\sup \left\{\frac{\limsup _{n} d\left(x_{n+1}, \operatorname{conv}\left(x_{j}\right)_{j=1}^{\infty}\right)}{A\left(\left(x_{n}\right)\right)}:\left(x_{n}\right)\right. \text { is a bounded }
$$

We have $M(X)=1 / W C S(X)$ for each reflexive Banach space $X$ and $M(X)=1$ for each nonreflexive Banach space $X$.

To formulate our results, we need to fix some notations. For any $i \in \mathbb{N}$, put

$$
\begin{aligned}
b_{i} & =\sup \left\{v>0: \Psi_{i}(v)<+\infty\right\}, \\
a_{i} & = \begin{cases}b_{i} & \text { if } \Psi_{i}\left(b_{i}\right)<1 \\
\Psi_{i}^{-1}(1) & \text { if } \Psi_{i}\left(b_{i}\right) \geq 1,\end{cases} \\
N_{x} & =\{i \in \mathbb{N}: x(i) \neq 0\} .
\end{aligned}
$$

The result that for every $x \in l^{\Phi}$ with $\sum_{i \in N_{x}} \Psi_{i}\left(a_{i}\right)>1$, we have $\|x\|=\frac{1}{k}\left(1+I_{\Phi}(k x)\right)$ if and only if $k \in\left[k_{x}^{*}, k_{x}^{* *}\right]$, where $k_{x}^{*}=\inf \left\{k>0: \sum_{i=1}^{\infty} \Psi_{i}\left(\varphi_{i}(k x(i))\right) \geq 1\right\}, k_{x}^{* *}=$ $\sup \left\{k>0: \sum_{i=1}^{\infty} \Psi_{i}\left(\varphi_{i}(k x(i))\right) \leq 1\right\}$ has been proved in [18]. Moreover, for any 
$x \in l^{\Phi}$ there exists $k>0$ such that $\|x\|=\frac{1}{k}\left(1+I_{\Phi}(k x)\right)$ whenever $\Phi_{i}(u) / u \rightarrow+\infty$ as $u \rightarrow+\infty$ for all $i \in \mathbb{N}$.

We need to define a regularity condition for $\Phi=\left(\Phi_{i}\right)$ called the $\delta_{2}^{0}$-condition. A Musielak-Orlicz function $\Phi=\left(\Phi_{i}\right)$ satisfies the $\delta_{2}^{0}$-condition if there exist positive constants $a$ and $K$ and a sequence $\left(c_{i}\right)$ in $[0,+\infty]$ such that $\sum_{i=i_{0}}^{\infty} c_{i}<+\infty$ for some $i_{0} \in \mathbb{N}$ and

$$
\Phi_{i}(2 u) \leq k \Phi_{i}(u)+c_{i}
$$

for each $i \in \mathbb{N}$ and each $u \in \mathbb{R}$ satisfying $\Psi_{i}(u) \leq a$. In the case when all $c_{i}$ are in $\mathbb{R}_{+}$we say that $\Phi$ satsfies the $\delta_{2}$-condition (see $[15]$ ).

\section{Results}

We start with the following

Lemma 1. If $\sum_{i=1}^{\infty} \Psi_{i}\left(a_{i}\right) \leq 1$ then $l^{\Phi}$ has the Schur property.

Proof. Suppose $x_{n}=\left(x_{n}(i)\right) \in S\left(l^{\Phi}\right)$ for each $n \in \mathbb{N}$ and $x_{n} \rightarrow x_{0}$ weakly. By $\sum_{i=1}^{\infty} \Psi_{i}\left(a_{i}\right) \leq 1$, we have $\left\|x_{n}\right\|=\sum_{i=1}^{\infty} a_{i}\left|x_{n}(i)\right|(n=1,2, \ldots)$. Define $z_{n}=$ $\left(a_{1} x_{n}(1), a_{2} x_{n}(2), \ldots\right)$ and $z_{0}=\left(a_{1} x_{0}(1), a_{2} x_{0}(2), \ldots\right)$. Then $z_{n} \in l^{1}$ for $n=$ $0,1, \ldots$ and $z_{n} \rightarrow z_{0}$ weakly in $l^{1}$ (because the weak convergence in $l^{\varphi}$ implies the weak convergence in $\left.l^{1}\left(\left(a_{i}\right)\right)\right)$. Since $l^{1}$ has the Schur property, we get $\left\|z_{n}-z_{0}\right\|_{l^{1}} \rightarrow$ 0 . Hence, in view of the equality

$$
\left\|x_{n}-x_{0}\right\|=\sum_{i=1}^{\infty} a_{i}\left|x_{n}(i)-x_{0}(i)\right|=\left\|z_{n}-z_{0}\right\|_{l^{1}},
$$

we get $\lim _{n}\left\|x_{n}-x_{0}\right\|=0$, i.e. $l^{\Phi}$ has the Schur property.

To calculate Maluta's coefficient for $l^{\Phi}$ we need to define some parameter for the generating Musielak-Orlicz function $\Phi=\left(\Phi_{i}\right)$.

In this definition there is some analogy to the definition of the parameter $d_{\varphi}$ in [7] (see also [17]).

For every $m, n \in \mathbb{N}$ and $k>1$, we define

$$
c(k, m, n)=\inf \left\{c_{k, x}>0: I_{\Phi}\left(\frac{k x}{c_{k, x}}\right)=\frac{k-1}{2} \text { and } x=\sum_{i=m}^{m+n} x(i) e_{i} \in S\left(l^{\Phi}\right)\right\} .
$$

The sequence $(c(k, m, n))_{n=1}^{\infty}$ is nonincreasing for every $k>1$ and $m \in \mathbb{N}$. Therefore, the limit

$$
d(k, m)=\lim _{n \rightarrow \infty} c(k, m, n)
$$

exists. Moreover, $d(k, m) \leq c(k, m, n)$ for every $k>1$ and $m, n \in \mathbb{N}$. The sequence $(d(k, m))_{m=1}^{\infty}$ is nondecreasing. Hence, the limit

$$
d_{k}=\lim _{m \rightarrow \infty} d(k, m)
$$

exists and $d_{k} \geq d(k, m)$ for every $k>1$ and $m \in \mathbb{N}$.

Define

$$
d(\Phi)=\inf \left\{d_{k}: k>1\right\} .
$$

We are now in a position to give the main result of the paper. 
Theorem 1. Assume that $\Phi=\left(\Phi_{i}\right)$ is a Musielak-Orlicz function such that all $\Phi_{i}(i=1,2, \ldots)$ are finitely valued and $\Phi_{i}(u) / u \rightarrow+\infty$ as $u \rightarrow+\infty$ for all $i \in \mathbb{N}$. Then: (i) If $l^{\Phi}$ is nonreflexive, then $M\left(l^{\Phi}\right)=1$; (ii) If $l^{\Phi}$ is reflexive, then $M\left(l^{\Phi}\right)=1 / d(\Phi)$.

Proof. Statement (i) follows immediately from the fact that $M(X)=1$ for every nonreflexive Banach space $X$. So, we only need to prove that $W C S\left(L^{\Phi}\right)=d(\Phi)$ whenever $l^{\Phi}$ is reflexive. It is well known that the reflexivity of $l^{\Phi}$ is equivalent to the fact that both $\Phi$ and $\Psi$ satisfy the $\delta_{2}^{0}$-condition.

First, we will prove that $W C S\left(l^{\Phi}\right) \leq d(\Phi)$. For each $\varepsilon>0$, by the definition of $d(\Phi)$, there is $k>1$ such that $d(\Phi)>d_{k}-\varepsilon$. Recall that $d_{k} \geq d(k, m)$ for all $k>1$ and $m \in \mathbb{N}$. By the definition of $d(k, m)$ there is $n(m) \in \mathbb{N}$ such that

$$
d(k, m)>c(k, m, n)-\varepsilon \quad \text { whenever } \quad n>n(m) .
$$

Finally, by the definition of $c(k, m, n)$ there exists $x_{m, n} \in S\left(l^{\Phi}\right)$ such that

$$
\begin{aligned}
& \text { (1) } c_{k, x_{m, n}}-\varepsilon<c(k, m, n), \quad x_{m, n}=\sum_{i=m}^{m+n} x_{m, n}(i) e_{i} \in S\left(l^{\Phi}\right), \\
& \text { (2) } I_{\Phi}\left(\frac{k x_{m, n}}{c_{k, x_{m, n}}}\right)=\frac{k-1}{2} .
\end{aligned}
$$

Take $m_{1}=1$. Then there exist $n_{1} \in \mathbb{N}, n_{1}>n\left(m_{1}\right)$ and $x_{m_{1}, n_{1}}$ satisfying (1) and (2) with $m_{1}$ and $n_{1}$ in place of $m$ and $n$. Take $m_{2}=m_{1}+n_{1}+1$. There exists $x_{m_{2}, n_{2}}$ satisfying (1) and (2) with $m_{2}, n_{2}, n_{2}>n\left(m_{2}\right)$, in place of $m, n$. By induction, we can construct a sequence $\left(x_{m_{i}, n_{i}}\right)_{i=1}^{\infty}$ in $S\left(l^{\Phi}\right)$ with pairwise disjoint supports and satisfying (1) and (2) with $m_{i}$ and $n_{i}, n_{i}>n\left(m_{i}\right)$ in place of $m$ and $n$ for $i=1,2, \ldots$.

Define $y_{k}=x_{m_{k}, n_{k}}$. Then, we have $y_{n} \in S\left(l^{\Phi}\right)$ for every $n \in \mathbb{N}$. Moreover, $y_{n} \rightarrow 0$ weakly and for every $\nu, l \in \mathbb{N}$ there holds

$$
\begin{aligned}
& \left\|\frac{y_{\nu}-y_{l}}{d(\Phi)+2 \varepsilon}\right\| \leq \frac{1}{k}\left(1+I_{\Phi}\left(k \frac{y_{\nu}-y_{l}}{d(\Phi)+2 \varepsilon}\right)\right)=\frac{1}{k}\left(1+I_{\Phi}\left(\frac{y_{\nu}}{d(\Phi)+2 \varepsilon}\right)+I_{\Phi}\left(\frac{y_{l}}{d(\Phi)+2 \varepsilon}\right)\right) \\
& \leq \frac{1}{k}\left(1+I_{\Phi}\left(\frac{k x_{m_{\nu}, n_{\nu}}}{c_{k, x_{m_{\nu}, n_{\nu}}}}\right)+I_{\Phi}\left(\frac{k x_{m_{l}, n_{l}}}{c_{k, x_{m_{l}, n_{l}}}}\right)\right) \\
& =\frac{1}{k}\left(1+\frac{k-1}{2}+\frac{k-1}{2}\right)=1 \text {. }
\end{aligned}
$$

Hence $A\left(\left(y_{n}\right)\right) \leq d(\Phi)+3 \varepsilon$. By the arbitrariness of $\varepsilon>0$, we have $A\left(\left(y_{n}\right)\right) \leq d(\Phi)$. By virtue of Proposition 2 in [19] which says that for each weakly convergent sequence on the unit sphere of a Banach space $X$ there exists an asymptotic equidistant subsequence, we can now conclude that $W C S\left(l^{\Phi}\right) \leq d(\Phi)$.

Next, we will prove that $W C S\left(l^{\Phi}\right) \geq d(\Phi)$. First, we will show the equality

$$
W C S\left(l^{\Phi}\right)=\inf \left\{A\left(\left(x_{n}\right)\right): x_{n}=\sum_{i=l_{n-1}+1}^{l_{n}} x_{n}(i) e_{i} \text { and }\left(x_{n}\right)\right.
$$

is an asymptotic equidistant sequence in $\left.S\left(l^{\Phi}\right)\right\}=: d$.

It is obvious that $W C S\left(l^{\Phi}\right) \leq d$, so we only need to prove that $W C S\left(l^{\Phi}\right) \geq d$. For any $\varepsilon>0$, by the definition of $W C S\left(l^{\Phi}\right)$, there exists a sequence $\left(x_{n}\right)$ in $S\left(l^{\Phi}\right)$ being an asymptotic equidistant sequence, weakly convergent to 0 and such that

$$
A\left(\left(x_{n}\right)\right)<W C S\left(l^{\Phi}\right)+\varepsilon .
$$


Let $v_{1}=x_{1}$. Take $l_{1} \in \mathbb{N}$ satisfying $\left\|\sum_{i=l_{1}+1}^{\infty} v_{1}(i) e_{i}\right\|<\varepsilon$. Such a number $l_{1}$ exists since by the reflexivity of $l^{\Phi}$, the generating function $\Phi=\left(\Phi_{i}\right)$ satisfies the $\delta_{2}^{0}$-condition. By $x_{n}(i) \rightarrow 0$ as $n \rightarrow \infty,\left(i=1,2, \ldots, l_{1}\right)$ there is $n_{0} \in \mathbb{N}$ such that

$$
\left\|\sum_{i=1}^{l_{1}} x_{n}(i) e_{i}\right\|<\varepsilon \text { whenever } n>n_{0} .
$$

Let us fix $N_{1}>n_{0}$ and set $v_{2}=x_{N_{1}}$. Then

$$
\left\|\sum_{i=1}^{l_{1}} v_{2}(i) e_{i}\right\|<\varepsilon
$$

Take $l_{2}>l_{1}$ such that $\left\|\sum_{i=l_{2}+1}^{\infty} v_{2}(i) e_{i}\right\|<\varepsilon$. By $x_{n}(i) \rightarrow 0$ as $n \rightarrow \infty$ for $i=$ $1,2, \ldots$, we can find $N_{2}>N_{1}$ such that

$$
\left\|\sum_{i=1}^{l_{2}} x_{n}(i) e_{i}\right\|<\varepsilon \text { whenever } n>N_{2} .
$$

Let us choose $N_{3}>N_{2}$ and set $v_{3}=x_{N_{3}}$. Then

$$
\left\|\sum_{i=1}^{l_{2}} v_{3}(i) e_{i}\right\|<\varepsilon
$$

Take $l_{3}>l_{2}$ such that

$$
\left\|\sum_{i=l_{3}+1}^{\infty} v_{3}(i) e_{i}\right\|<\varepsilon
$$

In such a way we can construct by induction a sequence $\left(l_{n}\right)$ of natural numbers with $l_{1}<l_{2}<\ldots$ and a subsequence $\left(v_{n}\right)$ of $\left(x_{n}\right)$ satisfying $A\left(\left(v_{n}\right)\right)=A\left(\left(x_{n}\right)\right)$ and

$$
\left\|\sum_{i=1}^{l_{n-1}} v_{n}(i) e_{i}\right\|<\varepsilon, \quad\left\|\sum_{i=l_{n}+1}^{\infty} v_{n}(i) e_{i}\right\|<\varepsilon
$$

where $l_{0}=0$ by definition. Put

$$
u_{n}=\sum_{i=l_{n-1}+1}^{l_{n}} v_{n}(i) e_{i} /\left\|\sum_{i=l_{n-1}+1}^{l_{n}} v_{n}(i) e_{i}\right\| \quad(n=1,2, \ldots) .
$$


Then $u_{n} \in S\left(l^{\Phi}\right)$ for each $n \in \mathbb{N}$. Moreover, for every $m, n \in \mathbb{N}, n<m$, we have

$$
\begin{aligned}
\left\|v_{n}-v_{m}\right\|= & \| \sum_{i=1}^{l_{n-1}}\left(v_{n}(i)-v_{m}(i)\right) e_{i}+\sum_{i=l_{n-1}+1}^{l_{n}}\left(v_{n}(i)-v_{m}(i)\right) e_{i} \\
& +\sum_{i=l_{n}+1}^{l_{m-1}}\left(v_{n}(i)-v_{m}(i)\right) e_{i}+\sum_{i=l_{m-1}+1}^{l_{m}}\left(v_{n}(i)-v_{m}(i)\right) e_{i} \\
& +\sum_{i=l_{m}+1}^{\infty}\left(v_{n}(i)-v_{m}(i)\right) e_{i} \| \\
\geq & \left\|\sum_{i=l_{n-1}+1}^{l_{n}} v_{n}(i) e_{i}-\sum_{i=l_{m-1}+1}^{l_{m}} v_{m}(i) e_{i}\right\|-4 \varepsilon \\
\geq & \left\|\left(u_{n}-u_{m}\right)(1-2 \varepsilon)\right\|-4 \varepsilon
\end{aligned}
$$

Therefore

$$
A\left(\left(u_{n}\right)\right) \leq \frac{A\left(\left(v_{n}\right)\right)}{1-2 \varepsilon}+\frac{4 \varepsilon}{1-2 \varepsilon}=\frac{A\left(\left(x_{n}\right)\right)+4 \varepsilon}{1-2 \varepsilon} \leq \frac{W C S\left(l^{\Phi}\right)+5 \varepsilon}{1-2 \varepsilon} .
$$

In view of the arbitrariness of $\varepsilon>0$, we have $d \leq W C S\left(l^{\Phi}\right)$. Finally, we will prove that $d \geq d(\Phi)$. For any asymptotic equidistant sequence

$$
x_{n}=\sum_{i=l_{n-1}+1}^{l_{n}} x_{n}(i) e_{i} \in S\left(l^{\Phi}\right) \quad(n=1,2, \ldots),
$$

there are $k_{m, n}>0$ such that

$$
\left\|\left(x_{m}-x_{n}\right) / d(\Phi)\right\|=\frac{1}{k_{m, n}}\left(1+I_{\Phi}\left(k_{m, n} \frac{x_{m}-x_{n}}{d(\Phi)}\right)\right)
$$

for all $m, n \in \mathbb{N}, m \neq n$. Let us assume in the following that $m, n \in \mathbb{N}$ and $m \neq n$. We will consider now two cases.

I. If $k_{m, n} \leq 1$, then $\left\|x_{m}-x_{n}\right\| \geq d(\Phi)$.

II. If $k_{m, n}>1$, then

$$
\begin{aligned}
\left\|\left(x_{m}-x_{n}\right) / d(\Phi)\right\| & =\frac{1}{k_{m, n}}\left(1+I_{\Phi}\left(\frac{k_{m, n} x_{m}}{d(\Phi)}\right)+I_{\Phi}\left(\frac{k_{m, n} x_{n}}{d(\Phi)}\right)\right) \\
& \geq \frac{1}{k_{m, n}}\left(1+\frac{k_{m, n}-1}{2}+\frac{k_{m, n}-1}{2}\right) \\
& =1
\end{aligned}
$$

whence we get again $\left\|x_{m}-x_{n}\right\| \geq d(\Phi)$. Consequently $A\left(\left(x_{n}\right)\right) \geq d(\Phi)$. By the arbitrariness of $\left(x_{n}\right)$ being an asymptotic equidistant sequence in $S\left(l^{\Phi}\right)$ it follows that $W C S\left(l^{\Phi}\right) \geq d(\Phi)$.

The following example shows how to compute $d(\Phi)$ in some concrete cases.

Example 1. Let $\Phi_{i}(u)=|u|^{p}$ for all $i \in \mathbb{N}$ and $u \in \mathbb{R}$, where $1<p<\infty$. If $\Phi=\left(\Phi_{i}\right)_{i=1}^{\infty}$, then $d(\Phi)=2^{1 / p}$.

Proof. It is obvious that $l^{\Phi}=l^{p}$. Moreover, $\|x\|=p^{1 / p} q^{1 / q}\|x\|_{p}$ for any $x \in l^{\Phi}$, where $1 / p+1 / q=1$ and $\|x\|_{p}=\left(\sum_{i=1}^{\infty}\left|x_{i}\right|^{p}\right)^{1 / p}$ (see [9]). Take arbitrary $k>1$ and $x \in S\left(l^{\Phi}\right)$ with finite support. It is easy to see that the number $c=c(k, x)>0$ 
satisfying the equality $I_{\Phi}\left(\frac{k x}{c}\right)=\frac{k-1}{2}$ is equal to $2^{1 / p} k(k-1)^{-1 / p} p^{-1 / p} q^{-1 / q}$. Therefore, $d(\Phi)=\inf \left\{2^{1 / p} k(k-1)^{-1 / p} p^{-1 / p} q^{-1 / q}: k>1\right\}$. To calculate this infimum it is enough to find $\inf \left\{k(k-1)^{-1 / p}: k>1\right\}$. Using the standard method, we get that this infimum is attained at $k_{0}=q$. Since $k_{0}-1=q / p$, we get $d(\Phi)=2^{1 / p}$.

\section{ACKNowledgement}

The authors thank the referee for valuable remarks.

\section{REFERENCES}

1. T. Dominguez Benavides, Weak uniform normal structure in direct-sum spaces, Studia Math. 103 (1992), 283-290. MR 94c:46024

2. T. Dominguez Benavides and G. Lopez Acedo, Lower bounds for normal structure coefficients, Proc. Roy. Soc. Edinburgh 121A (1992), 245-252. MR 93i:46025

3. T. Dominguez Benavides, G. López Acedo and Hong-Kun Xu, Weak uniform normal structure and iterative fixed points of nonexpansive mappings, Colloquium Math. 68 (1) (1995), 17-23. MR 95k:47086

4. W.L. Bynum, Normal structure coefficients for Banach spaces, Pacific J. Math. 86 (1980), 427-436. MR 81 m:46030

5. Yu.A. Cui, Midpoint locally uniform rotundity of Musielak-Orlicz sequence space with Orlicz norm, Northwestern J. Math. 9(4) (1993), 561-565. MR 96d:46017

6. K. Goebel and W.A. Kirk, Topics in Metric Fixed Point Theory, Cambridge University Press, 1990. MR 92c: 47070

7. H. Hudzik, C. Wu and Yi.N. Ye, Packing constant in Musielak-Orlicz sequence spaces equipped with the Luxemburg norm, Revista Math. 7 (1) (1994), 13-26. MR 95h:46013

8. W.A. Kirk, A fixed point theorem for mappings which do not increase distances, Amer. Math. Monthly 72 (1965), 1004-1006. MR 32:6436

9. M.A. Krasnoselskiı̌ and Ya.B. Rutickiı̌, Convex Functions and Orlicz Spaces (translation), Groningen 1961. MR 23:A4016

10. T. Landes, Permanence properties of normal structure, Pacific J. Math. 110 (1) (1984), 125143. MR 86e: 46014

11. T.C. Lim, On the normal structure coefficient and the bounded sequence coefficient, Proc. Amer. Math. Soc. 88 (1983), 262-264. MR 85g:46021

12. W.A.J. Luxemburg, Banach Function Spaces, Thesis, Delft 1955. MR 17:285a

13. L. Maligranda, Orlicz Spaces and Interpolation, Seminars in Math. 5, Campinas 1989.

14. E. Maluta, Uniformly normal structure and related coefficients for Banach spaces, Pacific J. Math. 111 (1984), 357-369. MR 85j:46023

15. J. Musielak, Orlicz Spaces and Modular Spaces, Lecture Notes in Math. 1034, Springer-Verlag 1983. MR 85m: 46028

16. M.M. Rao and Z.D. Ren, Theory of Orlicz Spaces, Marcel Dekker Inc., New York, Basel, Hong Kong 1991.

17. T.F. Wang, Packing sphere in Orlicz sequence spaces, Chinese Ann. Math. 6A: 5 (1985), $567-574$.

18. C.X. Wu and H.Yi. Sun, Norm calculation and complex convexity on sequence Musielak-Orlicz spaces, Chinese Ann. Math. 12A (1991), 98-102. MR 92h:46012

19. G.L. Zhang, Weakly convergent sequence coefficient of product space, Proc. Amer. Math. Soc. 117 (3) (1992), 637-643. MR 93d:46037

(Yunan Cui and Hongwei Zhu) Harbin University of Science and Technology, Department of Mathematics, Harbin (150080), China

E-mail address, Y. Cui: cuiya@hkd.hrbust.edu.cn

(Henryk Hudzik) Adam Mickiewicz University, Faculty of Mathematics and Computer Science, Matejki 48/49, 60-769 Poznań, Poland

E-mail address: hudzik@math.amu.edu.pl 